\title{
Finding all Convex Cuts of a Plane Graph in Cubic Time
}

Roland Glantz and Henning Meyerhenke 


\section{Fakultät für Informatik

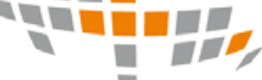

Please note:

This Report has been published on the Internet under the following Creative Commons License:

http://creativecommons.org/licenses/by-nc-nd/3.0/de. 


\title{
Finding all Convex Cuts of a Plane Graph in Cubic Time
}

\author{
Roland Glantz and Henning Meyerhenke \\ Institute of Theoretical Informatics \\ Karlsruhe Institute of Technology (KIT), Karlsruhe, Germany \\ Email: \{roland.glantz, meyerhenke\}@kit.edu
}

\begin{abstract}
In this paper we address the task of finding convex cuts of a graph. In addition to the theoretical value of drawing a connection between geometric and combinatorial objects, cuts with this or related properties can be beneficial in various applications, e.g., routing in road networks and mesh partitioning. It is known that the decision problem whether a general graph is $k$-convex is $\mathcal{N} \mathcal{P}$-complete for fixed $k \geq 2$. However, we show that for plane graphs all convex cuts (i. e., $k=2$ ) can be computed in polynomial time. To this end we first restrict our consideration to a subset of plane $\ell_{1}$-graphs for which the so-called alternating cuts can be embedded as plane curves such that the plane curves form an arrangement of pseudolines. For a graph $G$ in this set we formulate a one-to-one correspondence between the plane curves and the convex cuts of a bipartite graph from which $G$ can be recovered. Due to their local nature, alternating cuts cannot guide the search for convex cuts in more general graphs. Therefore we modify the concept of alternating cuts using the Djoković relation, which is of global nature and gives rise to cuts of bipartite graphs. We first present an algorithm that computes all convex cuts of a (not necessarily plane) bipartite graph $H^{\prime}=(V, E)$ in $\mathcal{O}\left(|E|^{3}\right)$ time. Then we establish a connection between convex cuts of a graph $H$ and the Djoković relation on a (bipartite) subdivision $H^{\prime}$ of $H$. Finally, we use this connection to compute all convex cuts of a plane graph in cubic time.
\end{abstract}

Keywords: Plane graphs, convex cuts, Djoković relation, partial cubes, bipartite graphs

\section{Introduction}

A convex cut of a graph $G=(V, E)$ is a partition of $V$ into $V_{1}$ and $V_{2}$ such that both subgraphs of $G$ induced by $V_{1}$ and $V_{2}$ are convex. A convex subgraph of $G$, in turn, is a subgraph $S$ of $G$ such that for any pair of vertices $v, w$ in $S$ all shortest paths from $v$ to $w$ in $G$ are fully contained in $S$. Following Artigas et al. [1], a convex $k$-partition in a graph is a partition of the vertex set into $k$ convex sets. If $G$ has a convex $k$-partition, then $G$ is said to be $k$-convex. Deciding whether a (general) graph is $k$-convex is $\mathcal{N} \mathcal{P}$-complete for fixed $k \geq 2$ [1]. Moreover, given a bipartite graph $G=(V, E)$ and an integer $k$, it is $\mathcal{N} \mathcal{P}$-complete to decide whether the largest convex set $\emptyset \neq S \subset V$ has at least $k$ vertices [8].

The fundamental notion of convexity in graphs can be used to draw a connection to continuous objects in a metric space. Note that there exists a different notion of convexity for plane graphs. A plane graph is called convex if all of its faces are convex polygons. This second notion is different and not object of our investigation. The notion of convexity in acyclic directed graphs, motivated by embedded processor technology, is also different [2]. There, a subgraph $S$ is called convex if there is no directed path between any pair $v, w$ in $S$ that leaves $S$. In addition to being directed, these paths do not have to be shortest paths as in our case.

Graph partitions with particular properties are of high interest in many applications [3]. Sample applications that potentially benefit from convexity of a cut are parallel numerical simulations. For some linear solvers used in these simulations, the shape of the partitions, in particular short boundaries, small aspect ratios, but also connectedness and smooth boundaries, plays a significant role [11]. Convex subgraphs typically admit these properties. Another example is the preprocessing of road networks for shortest path queries by partitioning according to natural cuts [6]. The definition of a natural cut is not as strict as that of a convex cut, but they have a related motivation.

Both from a theoretical point of view and due to the practical importance, it is natural to ask whether the time complexity of finding convex cuts is polynomial for certain types of inputs. In this paper, we focus on plane graphs as they are important in a large number of applications. Their special structure gives rise to the hope that finding their convex cuts is fundamentally easier. To the best of our knowledge, there exists no polynomial-time algorithm yet for computing convex cuts of plane graphs. 


\section{$1.1 \quad$ Related Work}

Artigas et al. [1] show that every connected chordal graph $G=(V, E)$ is $k$-convex, for $1 \leq k \leq|V|$. They also establish conditions on $|V|$ and $p$ to decide if the $p$ th power of a cycle is $k$-convex. Moreover, they present a linear-time algorithm that decides if a cograph is $k$-convex.

Our method for finding all convex cuts of a plane graph $G$ is motivated by the work in Chepoi et al. [5] on the links between alternating and convex cuts of plane graphs. Plane graphs usually have alternating cuts that are not convex and convex cuts that are not alternating. Proposition 2 in [5] characterizes the plane graphs for which the alternating cuts coincide with the convex cuts in terms of a condition on the boundary of any alternating cut. In this paper we represent the alternating cuts as plane curves that we call embedded alternating paths (EAPs) - any EAP partitions $G$ exactly like the alternating cut it represents. In contrast to [5], however, we focus on the intersections of the EAPs (i. e., alternating cuts).

If any pair of EAPs intersects at most once, we have a slight generalization of so-called arrangements of pseudolines. The latter arise in discrete geometry, computational geometry and in the theory of matroids [4]. Duals of arrangements of pseudolines are known to be partial cubes - a fact that has been applied to graphs in [9], for example. For basics on partial cubes we rely on the survey [12]. The following basic fact about partial cubes is crucial for our method for finding convex cuts: partial cubes are precisely the graphs that are bipartite and on which the Djoković relation [7] is transitive.

\subsection{Paper Outline and Contribution}

In Section 3 we first represent the alternating cuts of a plane graph $G=(V, E)$, as defined in [5], by EAPs. The main work here is on the proper embedding. We then study the case of $G$ being well-arranged, as we call it, i. e., the case in which the EAPs form an arrangement of pseudolines, or a slight generalization thereof. We show that the dual of such an arrangement is a partial cube, and that this partial cube contains another partial cube $\widetilde{G_{\mathcal{E}}}$ with the property that every other vertex on a path in $\widetilde{G_{\mathcal{E}}}$ has degree two. More importantly, $G$ can be regained from $\widetilde{G_{\mathcal{E}}}$ by fusing edges via degree-two vertices and deleting certain parallel edges. In particular, $G$ is an isometric subgraph of a half-cube, i.e., an $\ell_{1}$-graph, because for plane graphs this is equivalent [5]. We reveal a one-to-one correspondence between the EAPs of $G$ and the convex cuts of $\widetilde{G_{\mathcal{E}}}$. Specifically, the edges of $\widetilde{G_{\mathcal{E}}}$ intersected by any EAP form the cut-set of a convex cut of $\widetilde{G_{\mathcal{E}}}$. Conversely, the cut-set of any convex cut of $\widetilde{G_{\mathcal{E}}}$ is the set of edges intersected by a unique EAP of $G$.

The one-to-one correspondence between the EAPs of a well-arranged graph $G$ and the convex cuts of bipartite $\widetilde{G_{\mathcal{E}}}$ suggests that, for the case in which $G$ is more general, it might be helpful to employ a bipartite graph that can be turned into $G$ by contraction of degree-two vertices and reduction of parallel edges. We choose the simplest way to generate such a graph $G^{\prime}$, i.e., we subdivide any edge of $G$. Before we find all convex cuts of plane $G$ using $G^{\prime}$, we show in Section 4 that all convex cuts of a bipartite graph $G^{\prime}=\left(V^{\prime}, E^{\prime}\right)$ can be found in $\mathcal{O}\left(\left|E^{\prime}\right|^{3}\right)$ time, even if $G^{\prime}$ is not plane. The fact that we can compute all convex cuts in bipartite graphs in polynomial time is no contradiction to the $\mathcal{N} \mathcal{P}$-completeness of the decision problem whether the largest convex set in a bipartite graph has a certain size. In a convex cut both subgraphs have to be convex, while a convex set $S$ makes no assumptions on $V \backslash S$. The key to finding all convex cuts of a plane graph is a theorem that holds for non-plane graphs, too. In particular, we characterize convex cuts of $G$ in terms of the Djoković relation on $G^{\prime}$.

The results of Section 4 are then used in Section 5 to find all convex cuts of a plane graph $G=(V, E)$. As in the case of well-arranged graphs, we iteratively proceed from an edge to another edge opposite to a face bounded by $e$, and the number of such cuts is bounded by $2|E|$. This time, however, "opposite" is with respect to the Djoković relation on $G^{\prime}$. Thus we arrive at a set of potentially convex cuts, which includes all convex cuts. It remains to check whether the potentially convex cuts are actually convex. This can be done in $\mathcal{O}\left(|V|^{2}\right)$ time per potentially convex cut, totalling up to $\mathcal{O}\left(|V|^{3}\right)$ for all such cuts.

\section{Preliminaries}

Our methods for finding all convex cuts apply to finite, undirected, unweighted, and connected graphs that are free of self-loops and whose vertices all have degree greater than one. The last two conditions are not essential: self-loops have no impact on the convex cuts, and any edge on a dead-end path toward a degree-one 
vertex gives rise to a trivial convex cut that does not interfere with the non-trivial convex cuts. We often associate a cut $\left(V_{1}, V_{2}\right)$ with its cut-set, i. e., the set of edges running between $V_{1}$ and $V_{2}$.

If $G$ is plane, we may identify $V$ with a set of points in $\mathbb{R}^{2}$ and $E$ with a set of plane curves that intersect only at their end points, which, in turn, make up $V$. For $e \in E$ with end points $u, v(u \neq v)$ we sometimes write $e=\{u, v\}$ even when $e$ is not necessarily determined by $u$ and $v$ due to parallel edges. $F_{\infty}$ denotes the unbounded face of $G$ and, if $F$ is a face of $G$, we write $E(F)$ for the set of edges that bound $F$.

We denote the standard metric on $G$ by $d_{G}(\cdot, \cdot)$. In this metric the distance between $u, v \in V$ amounts to the number of edges on a shortest path from $u$ to $v$. A subgraph $S=\left(V_{S}, E_{S}\right)$ of a (not necessarily plane) graph $H$ is an isometric subgraph of $H$ if $d_{S}(u, v)=d_{H}(u, v)$ for all $u, v \in V_{S}$.

Following [7] and using the notation in [12], we set

$$
W_{x y}=\left\{w \in V: d_{G}(w, x)<d_{G}(w, y)\right\} \quad \forall\{x, y\} \in E .
$$

Let $e=\{x, y\}$ and $f=\{u, v\}$ be two edges of $G$. The Djoković relation $\theta$ on $G$ 's edges is defined as follows:

$$
e \theta f \Leftrightarrow f \text { has one end vertex in } W_{x y} \text { and one in } W_{y x} .
$$

The Djoković relation is reflexive, symmetric [14], but not necessarily transitive. The vertex set $V$ of $G$ is partitioned by $W_{a b}$ and $W_{b a}$ if and only if $G$ is bipartite.

A partial cube $G_{q}=\left(V_{q}, E_{q}\right)$ is an isometric subgraph of a hypercube. For a survey on partial cubes see [12]. Partial cubes and $\theta$ are related in that a graph is a partial cube if and only if it is bipartite and $\theta$ is transitive [12]. Thus, $\theta$ is an equivalence relation on $E_{q}$, and the equivalence classes are cut-sets of $G_{q}$. Moreover, the cuts defined by these cut-sets are precisely the convex cuts of $G_{q}$ [12]. If $\left(V_{q}^{1}, V_{q}^{2}\right)$ is a convex cut, the (convex) subgraphs induced by $V_{q}^{1}$ and $V_{q}^{2}$ are called semicubes. If $\theta$ gives rise to $k$ equivalence classes $E_{q}^{1}, \ldots E_{q}^{k}$, and thus $k$ pairs $\left(S_{a}^{i}, S_{b}^{i}\right)$ of semicubes, where the ordering of the semicubes in the pair is arbitrary, one can derive a so-called Hamming labeling $b: V_{q} \mapsto\{0,1\}^{k}$ by setting

$$
b(v)_{i}= \begin{cases}0 & \text { if } v \in S_{a}^{i} \\ 1 & \text { if } v \in S_{b}^{i}\end{cases}
$$

In particular, $d_{G_{q}}(u, v)$ amounts to the Hamming distance between $b(u)$ and $b(v)$ for all $u, v \in V_{q}$. This is a consequence of the fact that the corners of a hypercube have such a labeling and that $G_{q}$ is an isometric subgraph of a hypercube.

A half-cube $H$ is a graph whose vertices correspond to the vertices in one part of a bipartite representation of a partial cube $Q$. Two vertices of $H$ are adjacent in $H$ if their distance in $Q$ is two [5].

\section{Convex Cuts of a Class of Plane $\ell_{1}$-Graphs}

In Section 3.1 we define a multiset of (not yet embedded) alternating paths that are analogous to the alternating cuts defined in [5]. Section 3.2 is devoted to embedding the alternating paths into $\mathbb{R}^{2}$. In Section 3.3 we study the class of plane graphs that have embedded alternating paths (EAPs) which form an arrangement of pseudolines or a slight generalization thereof. For these so-called well-arranged graphs, the EAPs are equivalent to the convex cuts.

\subsection{Alternating Paths}

Intuitively, an EAP $P$ runs through a face $F$ of $G$ such that the edges through which $P$ enters and leaves $F$ are opposite - or nearly opposite because, if $|E(F)|$ is odd, there is no opposite edge, and $P$ has to make a slight turn to the left or to the right. The exact definitions leading up to (not yet embedded) alternating paths are as follows.

Definition 3.1 (Opposite edges, left, right, unique opposite edge).

Let $F \neq F_{\infty}$ be a face of $G$, and let $e, f \in E(F)$. Then $e$ and $f$ are called opposite edges of $F$ if the lengths of the two paths induced by $E(F) \backslash\{e, f\}$ differ by at most one. If the two paths have different lengths, $f$ is called the left [right] opposite edge of e if starting on e and running clockwise around $F$, the shorter [longer] path comes first. Otherwise, $e$ and $f$ are called unique opposite edges. 
Definition 3.2 (Alternating path graph $A(G)=\left(V_{A}, E_{A}\right)$ ).

The alternating path graph $A(G)=\left(V_{A}, E_{A}\right)$ of $G=(V, E)$ is the (non-plane) graph with $V_{A}=E$ and $E_{A}$ consisting of all two-element subsets $\{e, f\}$ of $E$ such that $e$ and $f$ are opposite edges of some face $F \neq F_{\infty}$.

The alternating path graph defined above will provide the edges for the multiset of alternating paths defined next. We resort to a multiset for the sake of uniformity, i.e., to ensure that any edge of $G$ is contained in exactly two alternating paths (see Figure 1a).

(a)

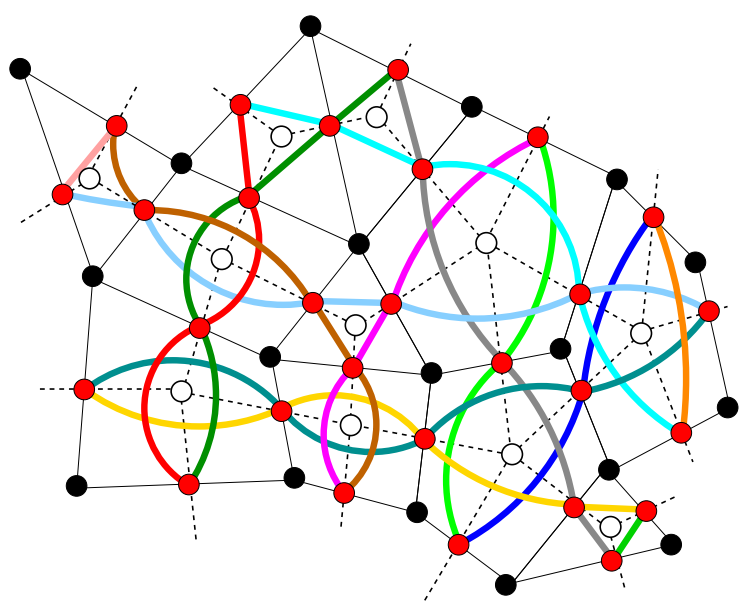

(b)

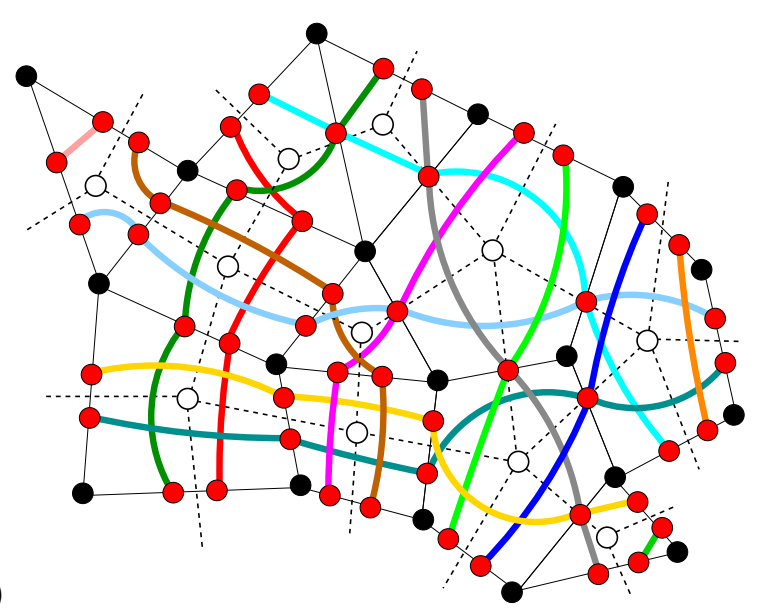

Fig. 1. Primal graph: Black vertices, thin solid edges. Dual graph: White vertices, dashed edges. (a) Multiset $\mathcal{P}(G)$ of alternating paths: Red vertices, thick solid lines. The paths in $\mathcal{P}(G)$ are colored. In this ad-hoc drawing all alternating paths that contain a vertex $v_{A}$ (edge $e$ of $G$ ) go through the same point on $e$, i. e., where a red vertex was placed. (b) Collection $\mathcal{E}(G)$ of EAPs: Red vertices, thick solid colored lines.

Definition 3.3 ((Multiset $\mathcal{P}(G)$ of) Alternating paths in $A(G)$ ). A maximal path $P=\left(v_{A}^{1}, e_{A}^{1}, v_{A}^{2}, \ldots e_{A}^{n-1}, v_{A}^{n}\right)$ in $A(G)=\left(V_{A}, E_{A}\right)$ is called alternating if

$-v_{A}^{i}$ and $v_{A}^{i+1}$ are opposite for all $1 \leq i \leq n-1$ and

- if $v_{A}^{i+1}$ is the left [right] opposite of $v_{A}^{i}$, and if $j$ is the minimal index greater than $i$ such that $v_{A}^{j}$ and $v_{A}^{j+1}$ are not unique opposites (and $j$ exists at all), then $v_{A}^{j+1}$ is the right [left] opposite of $v_{A}^{j}$.

The multiset $\mathcal{P}(G)$ contains all alternating paths in $A(G)$ : the multiplicity of $P$ in $\mathcal{P}(G)$ is two if $v_{A}^{i+1}$ is a unique opposite of $v_{A}^{i}$ for all $1 \leq i \leq n-1$, and one otherwise.

\subsection{Embedding of Alternating Paths}

In this section we show that the alternating paths in $\mathcal{P}(G)$ can be embedded into $\mathbb{R}^{2}$ such that any edge $\{e, f\}$ of any path in $\mathcal{P}(G)$ turns into a non-self-intersecting plane curve with one end point on $e$ and the other end point on $f$. Moreover, for any face $F$ of $G$ any pair of embedded paths intersects at most once in the filled polygon $\bar{F}=F \cup E(F)$. A path in $\mathcal{P}(G)$ with multiplicity $m \in\{1,2\}$ gives rise to $m$ embedded paths. Visually, we go from Figure 1a to Figure 1b.

In the following we set rules for embedding alternating paths locally, i. e., into $\bar{F}$. Later on we will make sure that the locally embedded paths fit together at the face boundaries. We formulate the rules only for faces whose boundaries are regular polygons. We may do so because all filled polygons in $\mathbb{R}^{2}$ (with the same number of sides) are homeomorphic - a consequence of the Jordan-Schönflies theorem [13]. Thus, we may embed the alternating paths first into a regular polygon $\overline{F_{r}}$ and then map the embedded paths from $\overline{F_{r}}$ into $\bar{F}$ using a homeomorphism from $\overline{F_{r}}$ onto $\bar{F}$. The homeomorphism will not affect the intersection pattern of the local embedding, i.e., information on which paths intersect in $F$ or hit an edge in $E(F)$. The intersection 
(a)

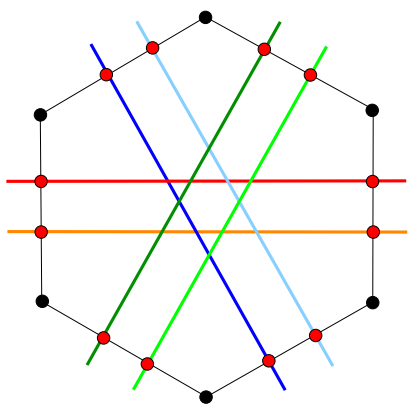

(b)

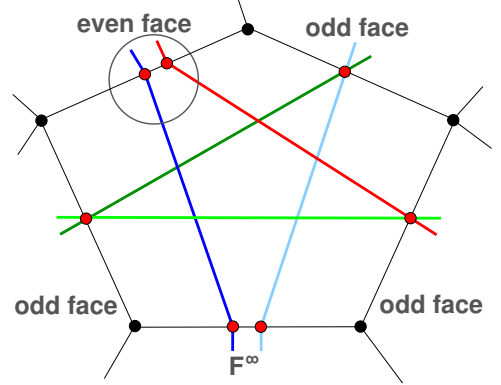

(c)

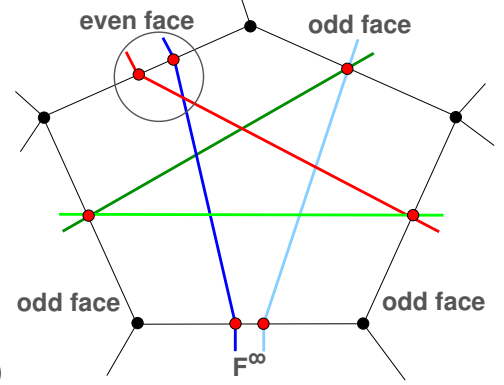

Fig. 2. (a) Intersection pattern of a filled hexagonal face (b,c) Two intersection patterns of a filled pentagonal face (see gray circle for difference).

pattern in $\overline{F_{r}}$ will depend not only on $\overline{F_{r}}$, but also on the cyclical order in which the EAPs from outside $\overline{F_{r}}$ hit $E(F)$. For examples see Figures 2b,c and Figure 3a.

Embedding Rules. We call a face $F$ of $G$ even [odd] if $|E(F)|$ is even [odd]. The rules for embedding alternating paths locally are:

1. The part of an EAP that runs through $F_{r}$ is a straight line, and EAPs cannot coincide in $F_{r}$.

2. An EAP can hit $e \in E\left(F_{r}\right)$ only at $e$ 's relative interior, i.e., not at $e$ 's end vertices.

3. If two EAPs share a point $p$ on an edge $e \in E\left(F_{r}\right)$, they must cross at $p$ and not just touch.

4. Let $F_{r} \neq F_{\infty}$ be an even face of $G$, let $e, f$ be unique opposite edges in $E\left(F_{r}\right)$, and let $P_{1}, P_{2}$ be the two non-embedded alternating paths in $\mathcal{P}(G)$ that contain the edge $\{e, f\}\left(P_{1}=P_{2}\right.$ if and only if the multiplicity of $P_{1}$ is two). Then the parts of embedded $P_{1}$ and $P_{2}$ that run through $\overline{F_{r}}$ must form a pair of distinct parallel line segments (see Figure 2a).

5. Let $F_{r} \neq F_{\infty}$ be an odd face of $G$, let $e \in E\left(F_{r}\right)$, and let $P_{1}, P_{2} \in \mathcal{P}(G)$ be the two paths that contain the vertex $e$. If $e$ also bounds an even bounded face, embedded $P_{1}, P_{2}$ must hit $e$ at two distinct points. If the other face is a bounded odd face, embedded $P_{1}, P_{2}$ must hit at a point on $e$. (see Figures $2 \mathrm{~b}, \mathrm{c}$ ).

6. Let $e$ be an edge of $G$ that separates $F_{\infty}$ from a bounded face, and let $P_{1}, P_{2} \in \mathcal{P}(G)$ be the two paths that contain the vertex $e$. Then, embedded $P_{1}, P_{2}$ must hit $e$ at two distinct points (see Figures $2 \mathrm{~b}, \mathrm{c}$ ).

We now map the embedded paths from any $\overline{F_{r}}$ into $\bar{F}$ using a homeomorphism from $\overline{F_{r}}$ onto $\bar{F}$. The following is about tying the loose ends of the locally embedded paths, which all sit on edges of $G$, so as to arrive at a global embedding of the alternating paths (see Figures 3b,c). Let $e \in E(G)$, and let $F, F^{\prime}$ be the faces of $G$ that are bounded by $e$. We have two locally embedded paths $P_{F}^{1}, P_{F}^{2}$ in $\bar{F}$ and two locally embedded paths $P_{F^{\prime}}^{1}, P_{F^{\prime}}^{2}$ in $\overline{F^{\prime}}$ that all hit $e$. If $F$ and $F^{\prime}$ are bounded odd faces, we bend the four paths such that they all hit the same point $q$ on the interior of $G$ (for details see below). Otherwise, let the end point of $P_{F}^{1}, P_{F}^{2}, P_{F^{\prime}}^{1}$, and $P_{F^{\prime}}^{2}$ be denoted by $p_{F}^{1}, p_{F}^{2}, p_{F^{\prime}}^{1}$, and $p_{F^{\prime}}^{2}$, respectively. Due to having used homeomorphisms to obtain the embedded paths in $\bar{F}$ and $\overline{F^{\prime}}$, it holds that $p_{F}^{1} \neq p_{F}^{2}$ and $p_{F^{\prime}}^{1} \neq p_{F^{\prime}}^{2}$. Let $e=\{u, v\}$, and let $q_{1}, q_{2}$ be points on the interior of $e$ such that $q_{1}$ is closer to $u$ than $q_{2}$. Without loss of generality we may assume that $p_{F}^{1}$ is closer to $u$ than $p_{F}^{2}$ and that $p_{F^{\prime}}^{1}$ is closer to $u$ than $p_{F^{\prime}}^{2}$. We now bend $p_{F}^{1}$ and $p_{F^{\prime}}^{1}\left[p_{F}^{2}\right.$ and $\left.p_{F^{\prime}}^{2}\right]$ toward $q_{1}\left[q_{2}\right]$.

It remains to show that the bending operations above can be done such that the intersection patterns do not change in the interiors of $F$ and $F^{\prime}$. Recall that the paths are homeomorphic to straight line segments. Thus, there exists $\epsilon>0$ such that all locally embedded paths in $F$ and $F^{\prime}$ other than $P_{F}^{1}, P_{F}^{2}, P_{F^{\prime}}^{1}$, and $P_{F^{\prime}}^{2}$ keep a distance greater than $\epsilon$ to $e$. The bending, in turn, can be done such that it affects $P_{F}^{1}, P_{F}^{2}, P_{F^{\prime}}^{1}$, and $P_{F^{\prime}}^{2}$ only in an $\epsilon$-neighborhood of $e$.

Notation 3.1. The collection of globally embedded alternating paths (EAPs) is denoted by $\mathcal{E}(G)$ (see again Figures 3b,c).

The EAPs in Figure 1b are special in that they form an arrangement in the following sense. 
(a)

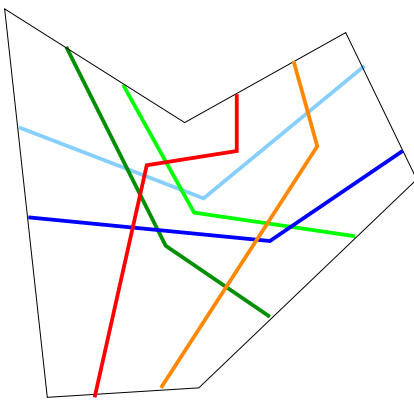

(b)

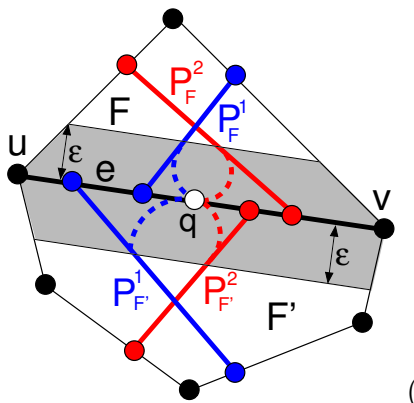

(c)

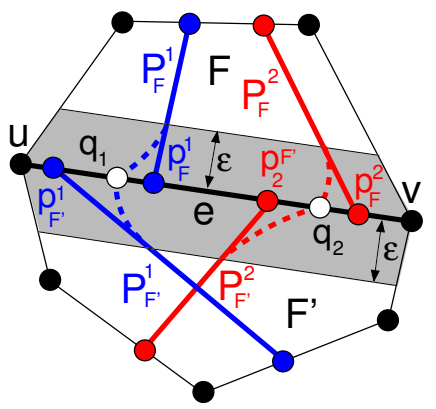

Fig. 3. (a) Hexagonal face with the same intersection pattern as in Figure 2a. (b,c) Bending of alternating paths is indicated by the dashed colored lines. For the notation see the text. (b) Bending toward a single point $q$ on $e$. (c) Bending toward two points $q_{1}, q_{2}$ on $e$.

Definition 3.4 (Arrangement of alternating paths, well-arranged graph). $\mathcal{E}(G)$ is called an arrangement of (embedded) alternating paths if

1. none of the EAPs is a cycle,

2. none of the EAPs intersects itself, and

3. there exist no paths $P_{1} \neq P_{2} \in \mathcal{E}(G)$ such that $P_{1} \cap P_{2}$ contains more than one point.

We call a plane graph $G$ well-arranged if $\mathcal{E}(G)$ is an arrangement of alternating paths.

The notion of an arrangement of alternating paths can be seen as a generalization of the notion of an arrangement of pseudolines [4]. The latter arrangements are known to have duals that are partial cubes [9].

\subsection{Embedded Alternating Paths of Well-arranged Graphs}

The purpose of the following is to prepare for the definition of a graph $G_{\mathcal{E}}$ (see Definition 3.6), which will turn out to be a partial cube if $G$ is well-arranged. In this case we are able to (i) efficiently compute the convex cuts of $G^{\mathcal{E}}$ and (ii) establish a one-to-one correspondence between the convex cuts of $G^{\mathcal{E}}$ and those of $G$.

Definition 3.5 (Domain $D(G)$ of $G$, facet of $\mathcal{E}(G)$, adjacent facets).

The domain $D(G)$ of $G$ is the set of points covered by the vertices, edges and bounded faces of $G$. A facet of $\mathcal{E}(G)$ is a (bounded) connected component (in $\left.\mathbb{R}^{2}\right)$ of $D(G) \backslash\left(\bigcup_{e \in E(G)} e \cup \bigcup_{v \in V(G)} v\right)$. Two facets of $\mathcal{E}(G)$ are adjacent if their boundaries share more than one point.

In the following DEAP stands for Dual of Embedded Alternating Paths.

Definition 3.6 (DEAP graph $G_{\mathcal{E}}$ of $G$ ).

A DEAP graph $G_{\mathcal{E}}$ of $G$ is a plane graph that we obtain from $G$ by placing one vertex into each facet of $\mathcal{E}(G)$ and drawing edges between a pair $(u, v)$ of these vertices if the facets containing $u$ and $v$ are adjacent in the sense of Definition 3.5. A vertex of $G_{\mathcal{E}}$ can also sit on the boundary of a face as long as it does not sit on an $E A P$ from $\mathcal{E}(G)$ (for an example see the black vertex on the upper left in Figure $4 a$ ).

Since we are not interested in the exact location [course] of $G_{\mathcal{E}}$ 's vertices [edges], there exists basically one DEAP graph of $G$. Due to the intersection pattern of the EAPs in $G$ 's bounded faces, as specified in Section 3.2 and illustrated in Figure 2, there are the following three kinds of vertices in $V\left(G_{\mathcal{E}}\right)$ :

\section{Definition 3.7 (Primal, intermediate and star vertex of $G_{\mathcal{E}}$ ).}

- Primal vertices: Vertices which represent a facet that contains a (unique) vertex $v$ of $G$ in its interior or on its boundary. As we do not care about the exact location of $G_{\mathcal{E}}$ 's vertices, we may assume that the primal vertices of $G_{\mathcal{E}}$ are precisely the vertices of $G$. 
(a)

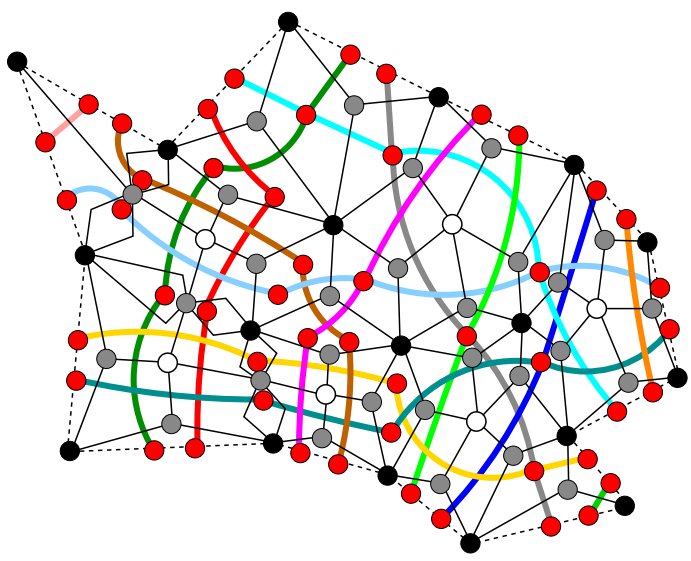

(b)

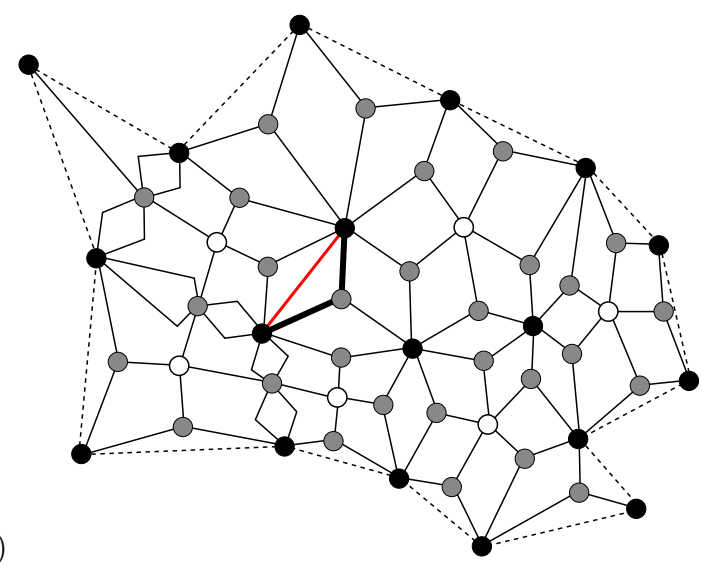

Fig. 4. DEAP graph $G_{\mathcal{E}}$ of the primal graph $G$ shown in Figure 1a. (a) Collection $\mathcal{E}(G)$ of EAPs: Red vertices, thick solid colored lines. DEAP graph $G_{\mathcal{E}}$ : Black, gray and white vertices, thin black solid lines. The black, gray and white vertices are the primal, intermediate and star vertices, respectively. The dashed polygonal line delimits $D(G)$. (b) $G \mathcal{E}$ only. The red edge, however, is an edge of $G$. The path formed by the two bold black edges is an example of a path in $G_{\mathcal{E}}$ of length two that connects two primal vertices that are adjacent in $G$ via an intermediate vertex in $G_{\mathcal{E}}$.

- Intermediate vertices: The neighbors of the primal vertices in $G_{\mathcal{E}}$.

- Star vertices The remaining vertices in $G_{\mathcal{E}}$.

For an example of a DEAP graph see Figure 4, where the black, gray and white vertices correspond to the primal, intermediate, and star vertices, respectively.

Theorem 3.1. The DEAP graph $G_{\mathcal{E}}$ of a well-arranged plane graph $G$ is a partial cube.

Proof. We denote the Hamming distance by $h(\cdot, \cdot)$. To show that $G_{\mathcal{E}}=\left(V_{\mathcal{E}}, E_{\mathcal{E}}\right)$ is a partial cube, it suffices to specify a labeling $l: V_{\mathcal{E}} \mapsto\{0,1\}^{n}$ for some $n \in \mathbb{N}$ such that $d_{G_{\mathcal{E}}}(u, v)=h\left(l(u), l(v)\right.$ ) for all $u, v \in V_{\mathcal{E}}$ (see Section 2).

We set the length $n$ of any binary vector $l(v)$ to the number of paths in $\mathcal{E}(G)$, and let the entries of $l(v)$ indicate $v$ 's position with respect to the paths in $\mathcal{E}(G)$. Specifically, we start by numbering the paths in $\mathcal{E}(G)$ from one to $n$, which yields the paths $P_{1}, \ldots, P_{n}$. For each $1 \leq i \leq n$ we then select one component of $D(G) \backslash P_{i}$. Then we set the $i$ th entry of $l(v)$ to one if the face represented by $v$ is in the selected component of $D(G) \backslash P_{i}$ (zero otherwise).

It remains to show that $d_{G_{\mathcal{E}}}(u, v)=h(l(u), l(v))$ for any pair $u \neq v \in V$. Since on any path of length $k$ from $u$ to $v$ in $G_{\mathcal{E}}$ it holds that $h(l(u), l(v)) \leq k$, we have $d_{G_{\mathcal{E}}}(u, v) \geq h(l(u), l(v))$.

To see that $d_{G_{\mathcal{E}}}(u, v)=h(l(u), l(v))$, it suffices to show that $u$ has a neighbor $u^{\prime}$ such that $h\left(l\left(u^{\prime}\right), l(v)\right)<$ $h(l(u), l(v))$ (because then there also exists $u^{\prime \prime}$ such that $h\left(l\left(u^{\prime \prime}\right), l(v)\right)<h\left(l\left(u^{\prime}\right), l(v)\right)$ and so on until $v$ is reached in exactly $h(l(u), l(v))$ steps). The claim follows from the case distinction in Section A.1 in the appendix.

The Hamming labelling of $G_{\mathcal{E}}$ defined in the proof of Theorem 3.1 guarantees the following.

Corollary 3.1. If $G$ is well-arranged, there exists a one-to-one correspondence between the EAPs of $G$ and the convex cuts of $G_{\mathcal{E}}$. Specifically, the edges intersected by any EAP of $G$ form a cut-set of a convex cut of $G_{\mathcal{E}}$. Conversely, the cut-set of any convex cut of $G_{\mathcal{E}}$ is the set of edges intersected by a unique EAP of $G$.

\subsection{Convex Cuts from Alternating Paths}

In case of $G$ being well-arranged, the subgraph defined below will serve as a stepping stone for relating the convex cuts of $G_{\mathcal{E}}$ to those of $G$. For an example see Figure 5. 


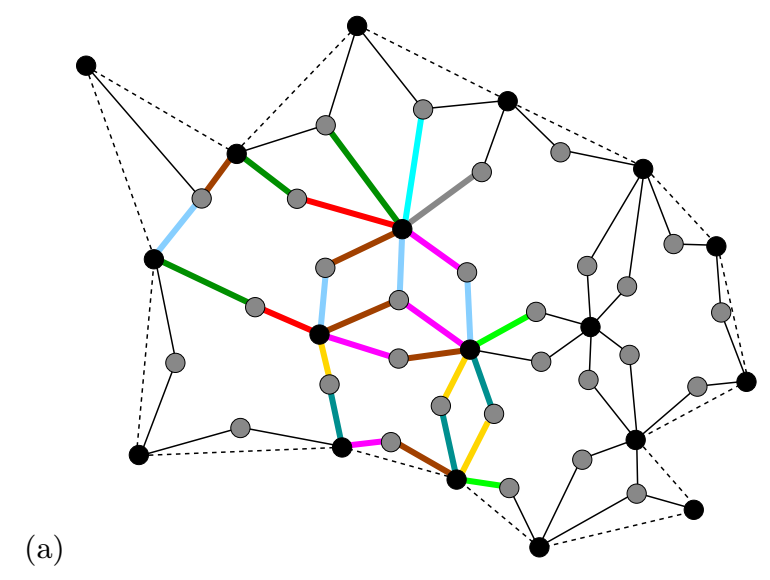

(b)

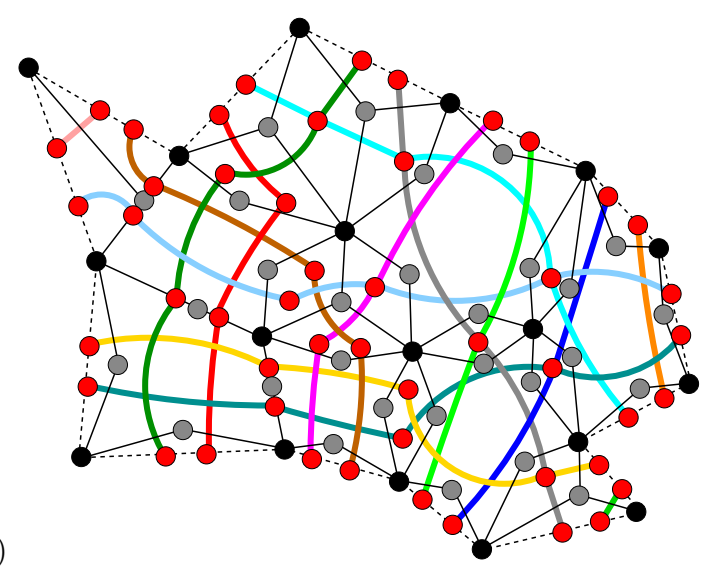

Fig. 5. (a) Isometric subgraph $\widetilde{G_{\mathcal{E}}}$ of the DEAP graph $G_{\mathcal{E}}$ shown in Figure $4 \mathrm{~b}$. The subgraph is obtained by deleting the star vertices of $G_{\mathcal{E}}$ and replacing parallel edges by single ones. A color of an edge is the color of the EAP that hits it. Here, the coloring is restricted to the edges in the vicinity of the brown cut-set. (b) EAPs from Figure $4 a$ drawn on the isometric subgraph shown on the left.

Definition 3.8 (Subgraph $\widetilde{G_{\mathcal{E}}}$ of $G_{\mathcal{E}}$ ).

$\widetilde{G_{\mathcal{E}}}$ is the graph obtained from $G_{\mathcal{E}}$ by deleting all star vertices and replacing parallel edges by single edges.

On the one hand $\widetilde{G_{\mathcal{E}}}$ is bipartite, every other vertex on a path being a vertex of $G$ (recall that we may interpret the primary vertices of $V\left(G_{\mathcal{E}}\right)$ as vertices of $G$ ). Thus, the distances between the primary vertices in $\widetilde{G_{\mathcal{E}}}$ are always twice the distances between the corresponding vertices in $G$. On the other hand, $\widetilde{G_{\mathcal{E}}}$ is an isometric subgraph of $G_{\mathcal{E}}$.

Lemma 3.1. $\widetilde{G_{\mathcal{E}}}$ is an isometric subgraph of $G_{\mathcal{E}}$.

Proof. For any face $F$ of $G$ let $G^{F}\left[G_{\mathcal{E}}^{F}\right]$ denote the subgraph of $G\left[G_{\mathcal{E}}\right]$ that is contained in $\bar{F}$. Any intersection pattern of $\bar{F}$ constitutes an arrangement of alternating paths of $G^{F}$, i.e., $G^{F}$ is well-arranged. Thus, Theorem 3.1 yields that $G_{\mathcal{E}}^{F}$ is a partial cube. In particular, the distance between any $u, v$ in $G_{\mathcal{E}}^{F}$ amounts to the minimum number of EAPs one has to cross on a path from $u$ to $v$. Let $\left(P_{1}, \ldots, P_{k}\right)$ be the sequence of paths crossed by a shortest path from $u$ to $v$, where $u$ and $v$ are vertices of $\widetilde{G_{\mathcal{E}}}$. The same sequence of $k$ paths is crossed in the same order, with no other paths in between, on the shorter of the two paths in $\widetilde{G_{\mathcal{E}}}$ that go around the star vertices in $F$ (see the intersection patterns).

So far we have seen that the distance between two vertices of $G_{\mathcal{E}}^{F}$ does not change when we delete the star vertices. Since any path on $\widetilde{G_{\mathcal{E}}}$ traverses adjacent faces of $G$, and since replacing parallel edges by single ones has no effect on the distances, we are done.

The first of the following corollaries is a straightforward extension of Corollary 3.1.

Corollary 3.2. If $G$ is well-arranged, there exists a one-to-one correspondence between the EAPs of $G$ and the convex cuts of $\widetilde{G_{\mathcal{E}}}$. Specifically, the edges intersected by any EAP of $G$ form a cut-set of a convex cut of $\widetilde{G_{\mathcal{E}}}$. Conversely, the cut-set of any convex cut of $\widetilde{G_{\mathcal{E}}}$ is the set of edges intersected by a unique EAP of $G$.

Corollary 3.3. A well-arranged graph is an $\ell_{1}$-graph.

Proof. Theorem 3.1 yields that $G_{\mathcal{E}}$ is a partial cube, and Lemma 3.1 then yields that $\widetilde{G_{\mathcal{E}}}$ is a partial cube, too. From $\widetilde{G_{\mathcal{E}}}$ being bipartite and the fact that one part consists of the primary vertices, i.e., vertices of $G$, we conclude that $G$ is a an isometric subgraph of a half-cube (see Section 2). The claim now follows from the fact that a plane graph is a partial half-cube if and only if it is an $\ell_{1}$-graph [5]. 
The EAPs of a general plane graph can have self-intersections, and a pair of EAPs can cross more than once. Thus, in such a more general setting, EAPs and the equivalent alternating cuts do not give rise to a one-to-one correspondence as in Corollary 3.2 and thus cannot be employed to find convex cuts. To solve this problem, we extend the concept of alternating cuts to graphs that are not necessarily plane by employing the Djoković relation on a (bipartite) subdivision of these graphs.

\section{Convex Cuts of not Necessarily Plane Graphs}

In this section we assume that $H^{\prime}=(V, E)$ is bipartite (but not necessarily plane). As mentioned in Section 2 , any edge $e=\{a, b\}$ of $H^{\prime}$ gives rise to a cut of $H^{\prime}$ into $W_{a b}$ and $W_{b a}$. The cut-set of this cut is $C_{e}=\{f \in E \mid$ $e \theta f\}=\left\{f=\{u, v\} \in E \mid d_{H^{\prime}}(a, u)=d_{H^{\prime}}(b, v)\right\}$. In the following we characterize the cut-sets of the convex cuts of $H^{\prime}$. This characterization is key to finding all convex cuts of a bipartite graph in $\mathcal{O}\left(|E|^{3}\right)$ time.

Lemma 4.1. Let $H^{\prime}=(V, E)$ be a bipartite graph, and let $e \in E$. Then $C_{e}$ is the cut-set of a convex cut of $H^{\prime}$ if and only if $f \theta \hat{f}$ for all $f, \hat{f} \in C_{e}$.

Proof. Let $C_{e}$ be a non-convex cut of $H^{\prime}$, and let $e=\{a, b\}$. Then there exists a shortest path $P=\left\{v_{1}, \ldots v_{n}\right\}$ with both end vertices in, say $W_{a b}$, such that $P$ has a vertex in $W_{b a}$. Let $i$ be the smallest index such that $v_{i} \in W_{b a}$, and let $j$ be the smallest index greater than $i$ such that $v_{j} \in W_{a b}$. Then $f=\left\{v_{i-1}, v_{i}\right\}$ and $\hat{f}=\left\{v_{j}, v_{j+1}\right\}$ are two edges in $C_{e}$. Lemma 3.5 in [12] says that no pair of edges from a shortest path are related by $\theta$, i. e., $f \theta \hat{f}$ does not hold.

Conversely, let $f=\{u, v\}, \hat{f}=\{\hat{u}, \hat{v}\} \in C_{e}$ such that $f \theta \hat{f}$ does not hold. Without loss of generality assume $\hat{u} \in W_{u v}, \hat{v} \in W_{v u}$ and $d_{H^{\prime}}(u, \hat{u})<d_{H^{\prime}}(v, \hat{v})$. Then $d_{H^{\prime}}(v, \hat{v})-d_{H^{\prime}}(u, \hat{u}) \geq 2$. Indeed, $d_{H^{\prime}}(v, \hat{v})-$ $d_{H^{\prime}}(u, \hat{u})$ must be even because otherwise one of the two distances $d_{H^{\prime}}(u, \hat{u})$ and $d_{H^{\prime}}(v, \hat{v})$ is even and the other one is odd. Hence, due to $H^{\prime}$ being bipartite and the fact that (adjacent) $u$ and $v$ are in different parts of $H^{\prime}$, the vertices $\hat{u}$ and $\hat{v}$ are in the same part of $H^{\prime}$, a contradiction to $\hat{u}$ and $\hat{v}$ being connected by $\hat{f} \in C_{e}$. From $d_{H^{\prime}}(v, \hat{v})-d_{H^{\prime}}(u, \hat{u}) \geq 2$ it follows that one can go from $v$ to $\hat{v}$, which are both in $W_{v u}$, by first traversing $f$, then going from $u$ to $\hat{u}$ within $W_{u v}$, and finally traversing $\hat{f}$. The length of this path is $\leq d_{H^{\prime}}(v, \hat{v})$. Thus, $C_{e}$ is not a cut-set of a convex cut of $H^{\prime}$.

Lemma 4.1 suggests to determine the convex cuts of $H^{\prime}$ as sketched in Algorithm 1.

Theorem 4.1. Algorithm 1 computes all convex cut-sets of a bipartite graph $H^{\prime}$ using $\mathcal{O}\left(|E|^{3}\right)$ time and $\mathcal{O}(|E|)$ space.

Proof. The correctness follows from Lemma 4.1 due to the symmetry of the Djoković relation. For each candidate cut-set, one needs to determine if all pairs of contained edges are Djoković related.

Regarding the running time, observe that for any edge $e$, the (not necessarily convex) cut-set $C_{e}$ can be determined by using BFS to compute the distances of any vertex to the end vertices of $e$. Hence each $C_{e}$ can be determined in time $\mathcal{O}(|E|)$. The inner for-loop has $\left.\mathcal{O}(|E|)\right)$ iterations. Each iteration has time complexity $\mathcal{O}(|E|)$ ) when using a linear-time set equality algorithm [10] (which requires linear space). Hence Algorithm 1 runs in $\mathcal{O}\left(|E|^{3}\right)$ time. Moreover, each cut-set is processed sequentially. Since no more than two cut-sets (with $\mathcal{O}(|E|)$ edges each) have to be stored at the same time, the space complexity of Algorithm 1 is $\mathcal{O}(|E|)$.

A simple loop-parallelization over the edges in line 3 would lead to a parallel running time of $\mathcal{O}\left(|E|^{2}\right)$ with $\mathcal{O}(|E|)$ processors. If one is willing to spend more processors and a quadratic amount of memory, then even faster parallelizations are possible. Since they use standard PRAM results, we forgo their description.

For the remainder of this section $H$ denotes a graph (self-loops and degree-one vertices allowed). We subdivide each edge of $H$ into two edges and thus get a bipartite graph $H^{\prime}$. An edge $e$ in $H$ that is subdivided into edges $e_{1}, e_{2}$ of $H^{\prime}$ is called parent of $e_{1}, e_{2}$, and $e_{1}, e_{2}$ are called children of $e$. The Djoković relation on $H^{\prime}$ is denoted by $\theta^{\prime}$.

The next lemma characterizes the convex cuts of $H$ in terms of the Djokovic relation on $H^{\prime}$. The lemma does, however, not imply that the convex cuts of $H$ can be derived from those of $H^{\prime}$ in polynomial time. 


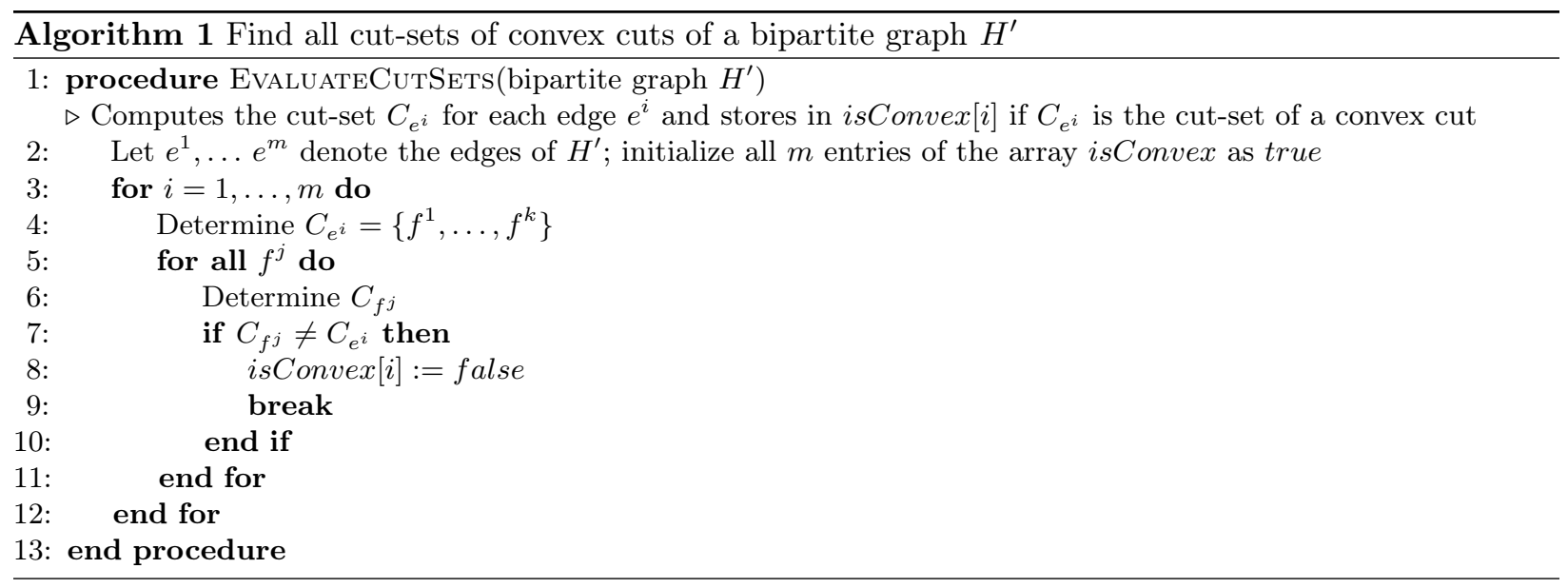

Lemma 4.2. A cut of $H$ with cut-set $C$ is convex if and only if for all $e, f \in C$ there exists a child $e^{\prime}$ of $e$ and a child $f^{\prime}$ of $f$ such that $e^{\prime} \theta^{\prime} f^{\prime}$.

Proof. Let $C$ be the cut-set of a convex cut that partitions $V$ into $V_{1}$ and $V_{2}$, and let $e, f \in C$ (see Figure 6a). We want to find a child $e^{\prime}$ of $e$ and a child $f^{\prime}$ of $f$ such that $e^{\prime} \theta^{\prime} f^{\prime}$. Let $e=\left\{u_{e}, v_{e}\right\}$ and $f=\left\{u_{f}, v_{f}\right\}$, and let $w_{e}^{\prime}\left[w_{f}^{\prime}\right]$ denote the vertex of $H^{\prime}$ that subdivides $e[f]$. Without loss of generality we assume $u_{e}, u_{f} \in V_{1}$ and $v_{e}, v_{f} \in V_{2}$. Since $C$ is the cut-set of a convex cut, we know that $d_{H}\left(u_{e}, u_{f}\right)$ and $d_{H}\left(v_{e}, v_{f}\right)$ differ by at most one. If $d_{H}\left(v_{e}, v_{f}\right)=d_{H}\left(u_{e}, u_{f}\right)$, let $e^{\prime}$ be the child of $e$ that has $u_{e}$ as an end vertex, and let $f^{\prime}$ be the child of $f$ that has $v_{f}$ as an end vertex (this is the case illustrated in Figure 6a). Then, due to the degrees of $w_{e}^{\prime}$ and $w_{f}^{\prime}$ being two, $d_{H^{\prime}}\left(u_{e}, w_{f}^{\prime}\right)=d_{H^{\prime}}\left(w_{e}^{\prime}, v_{f}\right)$, i.e., $e^{\prime} \theta^{\prime} f^{\prime}$. If $d_{H}\left(u_{e}, u_{f}\right)$ and $d_{H}\left(v_{e}, v_{f}\right)$ differ by exactly one, we may assume without loss of generality that $d_{H}\left(v_{e}, v_{f}\right)=d_{H}\left(u_{e}, u_{f}\right)+1$. Let, as above, $f^{\prime}$ be the child of $f$ that has $v_{f}$ as an end vertex, but let $e^{\prime}$ be the child of $e$ that has $v_{e}$ as an end vertex. Then, due to the degrees of $w_{e}^{\prime}$ and $w_{f}^{\prime}$ being two, $d_{H^{\prime}}\left(v_{e}, v_{f}\right)=d_{H^{\prime}}\left(w_{e}^{\prime}, w_{f}^{\prime}\right)$, i.e., $e^{\prime} \theta^{\prime} f^{\prime}$.

Conversely, let $C$ be the cut-set of a cut that partitions $V$ into $V_{1}$ and $V_{2}$. We now assume that for all $e=\left\{u_{e}, v_{e}\right\}, f=\left\{u_{f}, v_{f}\right\} \in C$ there exists a child $e^{\prime}$ of $e$ and a child $f^{\prime}$ of $f$ such that $e^{\prime} \theta^{\prime} f$. As above we assume without loss of generality that $u_{e}, u_{f} \in V_{1}$ and $v_{e}, v_{f} \in V_{2}$. There are four possibilities for the positions of $e^{\prime}$ and $f^{\prime}$ within $e$ and $f$, only two of which need to be considered due to symmetry.

1. $e^{\prime}=\left\{u_{e}, w_{e}^{\prime}\right\}$ and $f^{\prime}=\left\{u_{f}, w_{f}^{\prime}\right\}$. In this case $d_{H^{\prime}}\left(u_{e}, u_{f}\right)=d_{H^{\prime}}\left(w_{e}^{\prime}, w_{f}^{\prime}\right)=d_{H^{\prime}}\left(v_{e}, v_{f}\right) \pm 2$.

2. $e^{\prime}=\left\{u_{e}, w_{e}^{\prime}\right\}$ and $f^{\prime}=\left\{w_{f}^{\prime}, v_{f}\right\}$. Since the degrees of $w_{e}^{\prime}$ and $w_{f}^{\prime}$ are two, and since $e^{\prime} \theta^{\prime} f^{\prime}$, any shortest path from $u_{e}$ to $w_{f}^{\prime}$ runs via $u_{f}$, and any shortest path from $w_{e}^{\prime}$ to $v_{f}$ runs via $v_{e}$. Hence, $d_{H^{\prime}}\left(u_{e}, u_{f}\right)=d_{H^{\prime}}\left(u_{e}, w_{f}^{\prime}\right)-1=d_{H^{\prime}}\left(w_{e}^{\prime}, v_{f}\right)-1=d_{H^{\prime}}\left(v_{e}, v_{f}\right)$.

Thus $d_{H}\left(u_{e}, u_{f}\right)=d_{H}\left(v_{e}, v_{f}\right) \pm 1$ for all $e=\left\{u_{e}, v_{e}\right\}, f=\left\{u_{f}, v_{f}\right\} \in C$. Hence, any shortest path with end vertices in $V_{1}\left[V_{2}\right]$ stays within $V_{1}\left[V_{2}\right]$, i.e., $C$ is the cut-set of a convex cut.

\section{Convex Cuts of Plane Graphs}

Let $G=(V, E)$ denote a plane graph without self-loops and degree-one vertices. Analogous to Section 4, $G^{\prime}=\left(V^{\prime}, E^{\prime}\right)$ denotes the (plane bipartite) graph that one obtains from $G$ by placing a new vertex into the interior of each edge of $G$. For any face $F$ of $G$, we denote by $F^{\prime}$ the face of $G^{\prime}$ for which the parents of $E^{\prime}\left(F^{\prime}\right)$ form $E(F)$. The method for finding all convex cuts of $G$ in polynomial time that we present in this section is motivated by the following observations.

1. Using the Djoković relation, we can find all convex cuts of a bipartite graph in polynomial time, even if the graph is not plane (see Lemma 4.1).

2. A cut-set $C$ of a non-bipartite graph $H$ is the cut-set of a convex cut if and only if certain children of edges in $C$ are in the Djoković relation on $H^{\prime}$ (see Lemma 4.2). Again, $H$ does not need to be plane, 

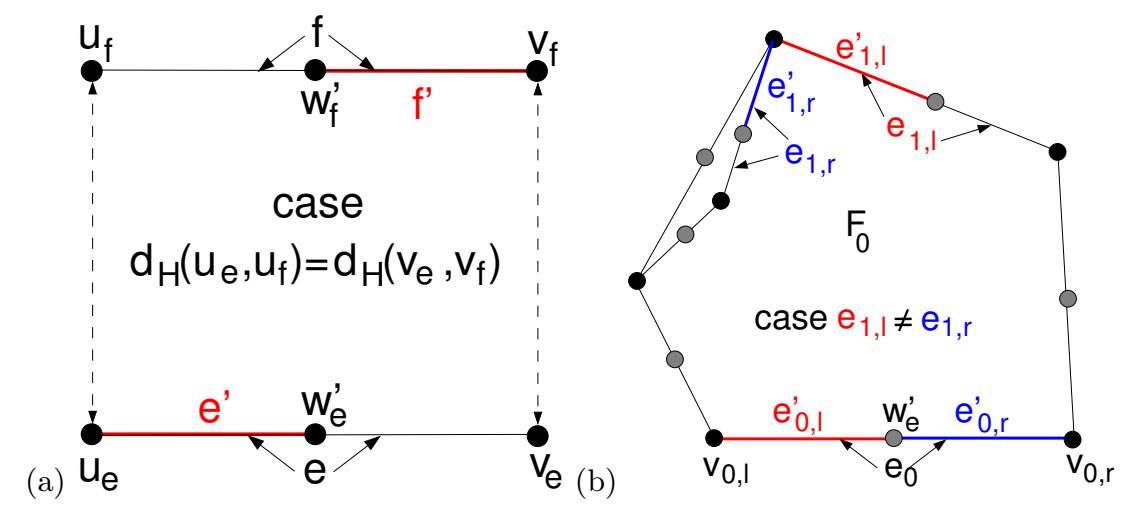

Fig. 6. (a) Illustration to proof of Lemma 4.2. (b) Cut-sets $\left\{e_{0}, e_{1, l}\right\}$ and $\left\{e_{0}, e_{1, r}\right\}$.

3. Let $C$ be the cut-set of a plane graph. Then one can brachiate through the edges of $C$ by forming a cyclic sequence $\left(e_{0}, \ldots e_{|C|-1}=e_{0}\right)$ such that for any pair $\left(e_{i}, e_{i+1}\right)$ of consecutive edges there exists a face $F$ with $\left\{e_{i}, e_{i+1}\right\} \in E(F)$ (indices are modulo $|C|$ ). Such a cyclic sequence, in turn, is equivalent to a cyclic sequence $\left(F_{0}, \ldots, F_{|C|-1}\right)$, where the (unique) $F_{i}$ are such that $\left\{e_{i}, e_{i+1}\right\} \in E\left(F_{i}\right)$. The cut-set $C$ is non-cyclical if and only if $F_{i}=F_{\infty}$ for some $i$.

In the following we will narrow down the search for convex cuts in $G$ by specifying cut-sets of potentially convex cuts, while making sure that the cut-set of any convex cut is among the specified cut-sets. We start with cut-sets that contain an edge $e_{0}$ bounding $F_{\infty}$. We also choose a face $F_{0} \neq F_{\infty}$ with $e_{0} \in E\left(F_{0}\right)$. Cases in which this is impossible are trivial. Below we will assemble cut-sets of potentially convex cuts, starting with $e_{0}$ and $F_{0}$. To do this, we need the following notation (see Figure $6 \mathrm{~b}$ ).

Let $e_{0, l}^{\prime}=\left\{v_{0, l}, w_{0}^{\prime}\right\}$ and $e_{0, r}^{\prime}=\left\{w_{0}^{\prime}, v_{0, r}\right\}$ be the two children of $e_{0}$. Here $v_{0, l}\left[v_{0, r}\right]$ is the left [right] vertex of $e_{0}$ when looking onto $e_{0}$ from the (unique) face $\neq F_{0}$ that bounds $e_{0}$, and $w_{0}^{\prime}$ is the vertex in $G^{\prime}$ that subdivides $e_{0}$. As in Section $4, C_{e^{\prime}}^{\prime}=\left\{f^{\prime} \in E^{\prime} \mid e^{\prime} \theta^{\prime} f^{\prime}\right\}$ is a cut-set of $G^{\prime}$ for all $e^{\prime} \in E^{\prime}$. In particular, $C_{e_{0, l}^{\prime}}^{\prime}$ $\left[C_{e_{0, r}^{\prime}}^{\prime}\right]$ contains two edges of $E^{\prime}\left(F^{\prime}\right)$, i. e., $e_{0, l}^{\prime}\left[e_{0, r}^{\prime}\right]$ and an edge denoted by $e_{1, l}^{\prime}\left[e_{1, r}^{\prime}\right]$. Finally, $e_{1, l}\left[e_{1, r}\right]$ is defined as the parent of $e_{1, l}^{\prime}\left[e_{1, r}^{\prime}\right]$.

Lemma 4.2 yields that, if the cut of $G$ with cut-set $C_{e_{0}}$ is convex, then $C_{e_{0}} \cap E\left(F_{0}\right)$ is equal to $\left\{e_{0}, e_{1, l}\right\}$ or $\left\{e_{0}, e_{1, r}\right\}$. It will turn out to be important that the sibling (child with same parent) of $e_{1, l}^{\prime}\left[e_{1, r}^{\prime}\right]$ is not related to $e_{0, r}^{\prime}\left[e_{0, l}^{\prime}\right]$ via $\theta^{\prime}$, even if the cut is not convex. This follows from the fact that $e_{1, l}^{\prime}$ and $e_{1, r}^{\prime}$ are unique. Thus, using Lemma 4.2 again, if $e_{1, l} \neq e_{1, r}$, there can be at most one cut-set of a convex cut that contains $e_{0}$ and $e_{1, l}\left[e_{0}\right.$ and $\left.e_{1, r}\right]$.

Analogous to the way in which $e_{0, l}^{\prime}\left[e_{0, r}^{\prime}\right]$ gives rise to $e_{1, l}^{\prime}\left[e_{1, r}^{\prime}\right], e_{1, l}^{\prime}\left[e_{1, r}^{\prime}\right]$ gives rise to $e_{2, l}^{\prime}\left[e_{2, r}^{\prime}\right]$, and so on, until some $e_{k, l}^{\prime}\left[e_{k, r}^{\prime}\right]$ bounds $F_{\infty}$ or coincides with some $e_{\hat{k}, l}^{\prime}\left[e_{\hat{k}, r}^{\prime}\right]$ with $\hat{k}<k$. Using Lemma 4.2 in each face $F_{i}$, as we have used it in $F_{0}$, yields the following.

Observation 5.1. If there exists a convex cut whose cut-set $C$ contains $e_{0}$, then $C=\left\{e_{0}, e_{1, l}, \ldots, e_{|C|-2, l}\right\}$ or $C=\left\{e_{0}, e_{1, r}, \ldots, e_{|C|-2, r}\right\}$.

Above we assumed that $e_{0}$ bounds $F_{\infty}$. Cut-sets of potentially convex cuts that do not involve edges bounding $F_{\infty}$ can then be found by starting at edges that do not bound $F_{\infty}$ and proceeding as above. Observation 5.1 then holds for all potentially convex cuts. In particular, for any edge $e$ of $G$ there exist at most two potentially convex cuts that both contain $e$. Thus, the total number of potentially convex cuts cannot exceed $2|E|$. Moreover, any potentially convex cut can be computed using $\mathcal{O}(|E|)$ time and $\mathcal{O}(|E|)$ space.

It remains to check whether a potentially convex cut is a convex cut. Due to Lemma 4.2 it suffices to perform the following for each edge $e$ of a cut-set $C$ of a potentially convex cut.

1. Let $e_{1}^{\prime}$ and $e_{2}^{\prime}$ denote the children of $e$. Determine $C_{e_{1}^{\prime}}=\left\{f^{\prime} \in E^{\prime} \mid e_{1}^{\prime} \theta^{\prime} f^{\prime}\right\}$ and $C_{e_{2}^{\prime}}=\left\{f^{\prime} \in E^{\prime} \mid e_{2}^{\prime} \theta^{\prime} f^{\prime}\right\}$. 
2. For all $f \neq e$ in $C$ check whether a child of $f$ is in $C_{e_{1}^{\prime}}$ or in $C_{e_{e}^{\prime}}$. If and only if at least one check fails, $C$ is not the cut-set of a convex cut.

Thus, as in Algorithm 1, checking whether one potentially convex cut is in fact convex, can be done using $\mathcal{O}\left(|E|^{2}\right)$ time. If the $\mathcal{O}(|E|)$ checks are performed successively, the space complexity is $\mathcal{O}(|E|)$. Since $G$ is plane, $\mathcal{O}(|E|)=\mathcal{O}(|V|)$. Also recall that the complications from self-loops and degree-one vertices can be solved in linear time (see Section 2). To summarize, we have shown the following.

Theorem 5.1. One can compute all convex cuts of a plane graph $G=(V, E)$ using $\mathcal{O}\left(|V|^{3}\right)$ time and $\mathcal{O}(|V|)$ space.

\section{Conclusions}

We have presented an algorithm for computing all convex cuts of a plane graph in cubic time. To the best of our knowledge, it is the first polynomial-time algorithm for this task. On the way to this result, we first represented alternating cuts as plane curves (EAPs) and focussed on a subset of $\ell_{1}$-graphs for which the EAPs basically form an arrangement of pseudolines. Thus we came across a one-to-one correspondence between the EAPs of a graph $G$ and the convex cuts of a bipartite graph $\widetilde{G_{\mathcal{E}}}$, one half of which is $G$. A similar correspondence on general graphs, in conjunction with an algorithm that computes all convex cuts of a bipartite graph in cubic time, formed the basis for our algorithm to compute all convex cuts of a general plane graph in cubic time, too. Consequently, while the problem is $\mathcal{N} \mathcal{P}$-hard for general graphs, we have shown that it becomes polynomial-time solvable for plane graphs. In future work we would like to investigate if the convexity test for potentially convex cuts can be accelerated asymptotically. It seems also worthwhile to apply the techniques based on the Djoković relation to other graph classes.

Acknowledgements. We thank Peter Sanders and Christian Schulz for helpful discussions on the topic.

\section{References}

1. D. Artigas, S. Dantas, M. Dourado, and J. Szwarcfiter. Partitioning a graph into convex sets. Discrete Mathematics, 311(17):1968 - 1977, 2011.

2. P. Balister, S. Gerke, G. Gutin, A. Johnstone, J. Reddington, E. Scott, A. Soleimanfallah, and A. Yeo. Algorithms for generating convex sets in acyclic digraphs. Journal of Discrete Algorithms, 7(4):509 - 518, 2009.

3. C. Bichot and P. Siarry. Graph Partitioning. Wiley, 2011.

4. A. Björner, M. Las Vergnas , B. Sturmfels, N. White, and G. Ziegler. Oriented Matroids. Encyclopedia of Mathematics and its Applications, 46. Cambridge University Press, second edition, 1999.

5. S. Chepoi. Clin d'œil on $L_{1}$-embeddable planar graphs. Discrete Applied Mathematics, 80:3 19, 1997.

6. D. Delling, A. V. Goldberg, I. Razenshteyn, and R. F. F. Werneck. Graph partitioning with natural cuts. In Proc. 25th IEEE Intl. Parallel and Distributed Processing Symposium (IPDPS'11), pages 1135-1146, 2011.

7. D. Djoković. Distance-Preserving Subgraphs of Hypercubes. Journal of Combinatorial Theory B, 14:263-267, 1973.

8. M. Dourado, F. Protti, D. Rautenbach, and J. Szwarcfiter. On the convexity number of graphs. Graphs and Combinatorics, 28:333-345, 2012.

9. D. Eppstein. Cubic Partial Cubes from Simplicial Arrangements. The electronic journal of combinatorics, 13:\#R79, 2006.

10. I. Katriel. On the algebraic complexity of set equality and inclusion. Information Processing Letters, 92(4):175 $-178,2004$.

11. H. Meyerhenke, B. Monien, and T. Sauerwald. A new diffusion-based multilevel algorithm for computing graph partitions. Journal of Parallel and Distributed Computing, 69(9):750-761, 2009. Best Paper Awards and Panel Summary: IPDPS 2008.

12. S. Ovchinnikov. Partial cubes: Structures, characterizations, and constructions. Discrete Mathematics, 308:5597$5621,2008$.

13. C. Thomassen. The Jordan-Schönflies Theorem and the Classification of Surfaces. The American Mathematical Monthly, 99:116-130, 1992.

14. E. Wilkeit. Isometric Embeddings in Hamming Graphs. Journal of Combinatorial Theory B, 14:179-197, 1990. 


\section{A Appendix}

\section{A.1 Proof Remainder of Theorem 3.1}

Proof. Let $F_{u}$ denote the facet of $\mathcal{E}(G)$ that is represented by $u$, and $I(u)$ denote the set of indices of paths in $\mathcal{E}(G)$ that bound $F_{u}$.

1. If $u$ has only one neighbor $u^{\prime}$, then $I(u)=\{k\}$ for some $k$, and the only vertex in one of the components of $D(G) \backslash P_{k}$ is $u$. For an example see the black vertex in the upper left corner of Figure 4b. Since $l(u)$ and $l\left(u^{\prime}\right)$ differ only at position $k$, it must hold that $h\left(l\left(u^{\prime}\right), l(v)\right)<h(l(u), l(v))$.

2. If $u$ has at least two neighbors, we first assume that none of the $P_{k}$ with $k \in I(u)$ cross each other (see Figure 7a). Then $u$ is uniquely determined by the entries of $l(u)$ at the positions given by $I(u)$. Indeed, $F_{u}$ is then bounded by non-intersecting and non-self-intersecting paths in $\mathcal{E}(G)$ that go from a point on the border of $D(G)$ to another point on the border of $D(G)$. Hence only a vertex inside $F_{u}$ can have the same entries in $l(\cdot)$ as $l(u)$ at the positions given by $I(u)$. Thus, since $u$ is the only vertex in $F_{u}$ and since $u \neq v, l(u)$ and $l(v)$ must differ at a position in $I(u)$, and we are done.

3. The remaining case is that $u$ has at least two neighbors and there exists at least one pair $(i, j) \in$ $I(u) \times I(u), i \neq j$, such that $P_{i}$ crosses $P_{j}$. Let $C$ denote the set of all such pairs. For any pair $(i, j) \in C$ the path $P_{i}$ crosses the path $P_{j}$ exactly once, because $\mathcal{E}(G)$ is an arrangement of alternating paths (see Figure $7 \mathrm{~b}$ ). Thus $P_{i}$ and $P_{j}$ subdivide $D(G)$ into four regions, each of which is characterized by one of the four $0 / 1$ combinations of vertex label entries at $i$ and at $j$. We may assume that $v$ is contained in the same region as $u$ for each pair $(i, j) \in C$ (otherwise we choose $u^{\prime}$ on the other side of $P_{i}$ or $P_{j}$ and are done). The intersection of all these regions, one region per pair in $C$, is denoted by $R$.

If all pairs $(i, j) \in I(u) \times I(u), i \neq j$, are in $C$, we are done. Indeed this means that $R=F_{u}$ and thus that $u$ is uniquely determined by the entries of $l(u)$ at the positions given by $I(u)$. We can then proceed as above. The remaining case is that there exist $i \in I(u)$ such that $P_{i}$ does not intersect any $P_{j}$ with $j \in I(u), j \neq i$ (see Figure $7 \mathrm{c}$ ). Let the set of these indices be denoted by $I^{\prime}(u)$. In particular the facets of $\mathcal{E}(G)$ that are contained in $R$ are separated by the paths $P_{i}$ with $i \in I^{\prime}(u)$. Recall that we assumed $u \neq v, u, v \in R$, i.e., $u$ and $v$ are contained in different facets of $R$. Since the paths $P_{i}$ with $i \in I^{\prime}(u)$ do not cross each other, the entries of $l(u)$ and $l(v)$ differ at all positions in $I(u)$, and $u^{\prime}$ with $h\left(l\left(u^{\prime}\right), l(v)\right)<h(l(u), l(v))$ can be reached from $u$ by crossing a single path $P_{i}$ with $i \in I^{\prime}(u)$.

(a)

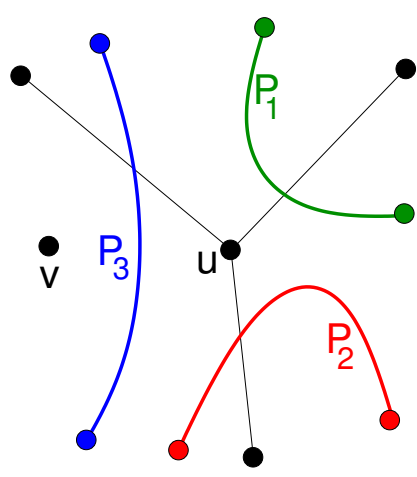

(b)

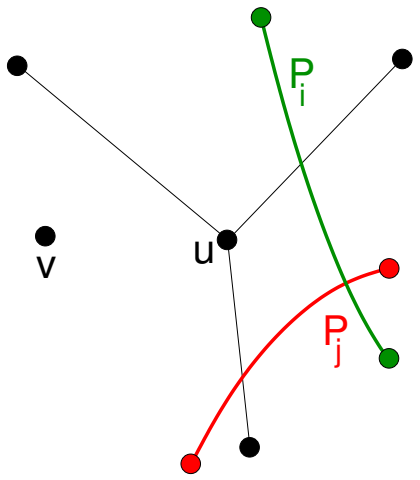

(c)

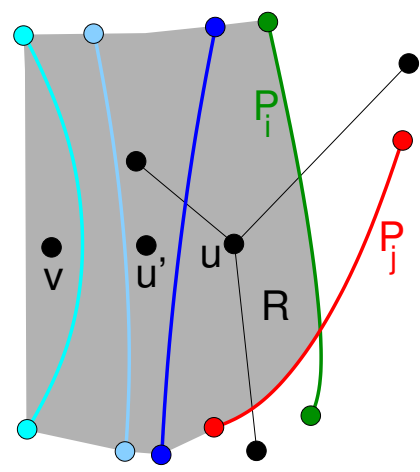

Fig. 7. (a) Illustration to item 2) in proof of Theorem 3.1. (b,c) Illustrations to item 3) in proof of Theorem 3.1. The shaded facet in (c) indicates $R$. 